\title{
Research on Hydrostatic Thrust Bearing Worktable Job Status
}

\author{
Jian-yi Zhang ${ }^{1}$, Xuan-Yu Shi ${ }^{2}$ and Gui-Hua Han ${ }^{1,3^{*}}$ \\ ${ }^{1}$ College of Mechanical \& Power Engineering, Harbin University of Science and \\ Technology, Harbin, China \\ ${ }^{2}$ Oil Product No. 4, Daqing Oilfield Company Ltd., Daqing 163511, China \\ ${ }^{3}$ Qiqihar Heavy CNC Equipment Corp. LTD. Qiqihar Heilongjiang Province, China \\ *641544105@qq.com
}

\begin{abstract}
The idea of the hydrostatic thrust bearing pose control was presented to mprove NC machine tool precision. The bearing worktable poses by controlled by coupling oil film thickness of 4 controllable chambers. The chamber flow can be regulated by ariable pump. The flow of the variable pump was controlled by electro hydrautic servo yalve according to the surface roughness, load, cutting force, and thermal effects of worktable. The mathematical models of the controllable chamber flow. The pose control model was established, which contains the kinematics positive and negative solution. The simulation experiment was carried out on the electro hydraulic servo test bench. The experiment results show that the model of hydrostatic thrust beoring pose is reasonable and control scheme is effective.
\end{abstract}

Keywords: hydrostatic thrust bealng; worktable pose; controllable chamber

\section{Introduction}

The hydrostatic thrust bearing are widely used in the heavy equipment because of the big bearing capacity and stable and reliable running. It is important parts of heavy vertical lathe. The research on hydrostatic thrust bearing mainly concentrates on the design of structure and the fubricating properties of the thrust bearing system Recess shape on the perormance of a capillary compensated circular thrust pad bearing were studied in documents [13], and different restrictors were studied comparatively of hydrostatic thrust bearing [4]. The simultaneous effects of inertia and temperature on the performance were analyzed [5]; C. K. Singh and D.V. Singh optimized stiffness of circular cavity tile and the rectangular tile of a variable restrictor-compensated hydrostatic thrust bearing [6]. T. Jayachandra Prabhu and N. Ganesan has analyzed that thrust plate incline and revolving impact on the rigidity of the multipad hydrostatic thrust bearing, and carried on the rigidity performance contrast experiment with that of the annular chamber [7-9]. But the research on the thrust bearing for quantitative oil supply system is few, and concentrates on theoretical analysis and numerical simulation, especially subject to the changes in external load, cutting force, thermal effect, surface roughness, and other factors.

On the heavy hydrostatic thrust bearing the open-loop control quantitative oil supply system are adopted generally. In quantitative oil supply system the motor drives the oil pump at constant rotational speed, the oil pump provides the constant flow for various oil separators, oil film thickness cannot be adjusted in the process. On the heavy hydrostatic thrust bearing the open-loop control quantitative oil supply system are 
adopted generally, in which the motor drives the oil pump at constant rotational speed, the oil pump provides the constant flow for various oil separators, oil film thickness cannot be adjusted in the process.

In the actual working environment, the oil viscosity and the oil temperature change according to room temperature, rotational speed etc. The open-loop control quantitative oil supply system are adopted generally on the heavy hydrostatic thrust bearing, in which the motor drives the oil pump at constant rotational speed, the oil pump provides the constant flow for various oil separators, oil film thickness cannot be adjusted in the process. In the actual working environment, the oil temperature changes according to room temperature, rotational speed etc, the oil viscosity also changes. The viscosity drops while temperature increases, and viscosity rises when the temperature reduces, simultaneously the change of the weight (load) of the work-piece in process is sometimes greater. Therefore, the film thickness will deviate from optimum value due to the time-variable oil viscosity and the load, which will destroy the working performance of hydrostatic bearing, simultaneously reduce the NC machine tool machining precision and working range.

A concept of controllable chambers is presented. The chambers include the constant flow chambers and the controllable flow chambers. The electro-hydraulic servo valve is used to overcome effectively the unbalance force and other disturbance force through 4 controllable chambers supplying oil cooperation, which can control the pose of bearing worktable, realize the high precision of spinde rotation

\section{Controllable Chambers}

The hydrostatic thrust bearing is composed of the worktable and the base. There are 30 chambers on the base, 26 of themare constant flow chambers, and the other 4 are the controllable flow chambers. There is a fixed gap between the work table and the base, and stable flow is supplied by the hydraulic pump to each constant flow chamber through the oil separator, the variable flow is supplied by the controllable chamber alone. The oil film in the fixed gap with certain rigidity can support and lubricate the bearing.

When the bearing displacement changes because of the external load, the changes in film thickness are detected by the electric eddy sensors, and feed-backed to the PLC; the control algorithm is completed and control signal is outputted by the PLC; electrohydraulic servo valve electric current signal is adjusted through the power amplifier; then the hydraulio cylinder and the inclination angle of the slanting plate of variable pump are controlled, the flow of the variable pump that outputted to the controllable chamber can'self-regulate along with the changes of the external load, so the oil film thickness maintains invariable, the rigidity and precision of bearings are improved, shown in Figure 1.

\section{Worktable Pose Model}

The supporting capacity of the bearing is obtained by changing the pressure distributing of the flow field. The traditional quantitative oil hydrostatic bearing supplies quantitative oil to chambers by invariable pump, when the supplying oil pressure $p_{p}$ is invariable, the supporting capacity is produced by changing the oil film thickness. Therefore, when external load appeared, the oil film thickness of the bearing would change by the variable work table displacement to resist external load. This will cause the position precision declined. This paper adopts the way of combining the 
constant and controllable chambers, When 4 controllable chambers are working, the oil film thickness is assured with no change, but the pressure field distribution can change to adapt the external load change in order to enhance the bearing rigidity. The constant chambers play master auxiliary support role, the chamber flow is constant. When the external force does not exist or the external force is very small, the controllable chambers may stop the work. At this time, the constant chamber mainly undertakes the support function. When the external force or unbalance bearing force exists, the controllable chambers and the constant chambers will use together, while the function of constant chambers is to support bearing by certain pressure field distribution, the function of controllable chambers is to resist the change of outside load and improve bearing rigidity by adjusting pressure field distribution actively. Through four controllable chambers supply oil in phase, the worktable may realize the vertical direction translation, three direction rotations as well as their compound motion separately in the space. The four contact points (controllable chamber) in base is located in circumference, whose radius is $r, B_{i}(i=1,2,3,4)$ is uniformly distributed in circumference. Four contact points on the worktable are lôcated in circumference, whose radius is $r, A_{i}(i=1,2,3,4)$ is uniformly distributed in circumference. The origin of body coordinate system $P_{x y z}$ is selected as circumcirclecenter of the top contact point circumcircle, and is fixed on the worktable. The static coordinate system $O_{x y z}$ is fixed on the base, and body coordinate system and the static coordinate system are superpositioned in initial point. The worktable initial beight, film thickness set is $\mathrm{h}$ (vertical distances between point $P$ and point $O$ ) In the bearing running process, it is must be guaranteed $B_{i}=A_{i}$ throughout. Coordinate axis direction is shown in Figure 2.

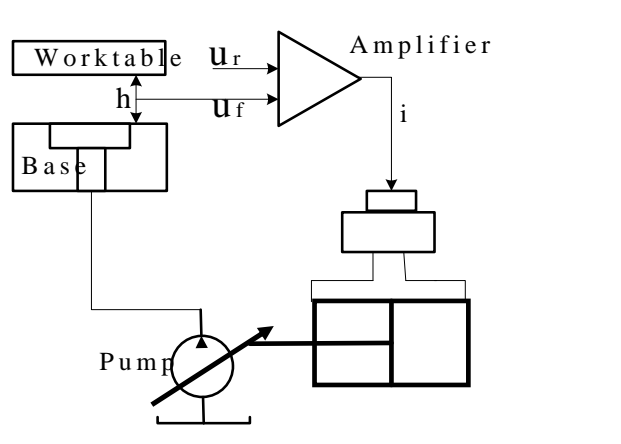

Figure 1. Schematic of Hydrostatic Thrust Bearing Controllable Chamber

\section{Mathematical Model of Bearing System}

\subsection{Chamber Equation Flow}

The thrust bearing with the annular chamber is shown in Figure 3, where the lubricating oil enters the annular chamber, then flows outward with scattering shape [10]. The flow of the thrust bearing with the annular chamber is:

$$
\begin{gathered}
Q_{1}=B p_{r} h^{3} / \mu \\
B=\pi / 6\left[1 / \ln \left(R_{4} / R_{3}\right)+1 / \ln \left(R_{2} / R_{1}\right)\right]
\end{gathered}
$$


Where, $B$ is coefficient of supporting flow; $p_{r}$ is chamber pressure, because the flow of hydraulic pump goes straight to the supporting chamber, circuit losses is neglected, the output pressure of the hydraulic pump is equal to the pressure of the chamber $p_{r}\left(\mathrm{~N} / \mathrm{m}^{2}\right) ; \mu$ is the dynamic viscosity of hydraulic oil $\left(\mathbb{N} \cdot \mathrm{s} / \mathrm{mm}^{2}\right) ; h$ is the oil film thickness(m).

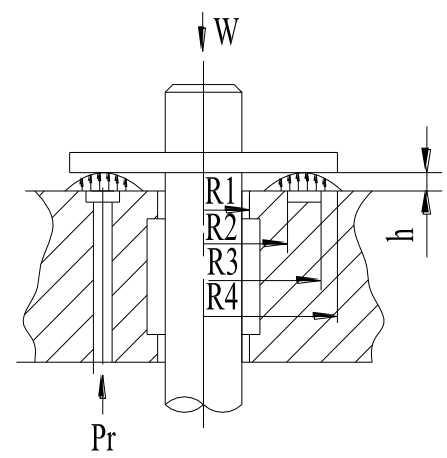

Figure 3. Annular Chamber of Hydrostatic Thrust Bearing

$$
p_{r}=W / A_{e}
$$

Where $W$ is the effective load, including the weight of the work-piece and the weight of the work table $(\mathrm{N}), A_{e}$ is the effective supporting area of the thrust bearing with the annular chamber $\left(\mathrm{m}^{2}\right)$.

$$
\begin{aligned}
& A_{34}=(\pi / 2)\left(R_{4}^{2}-R_{3}^{2}\right) / \ln \left(R_{4} / R_{3}\right) \\
& R_{12}=\left(R_{2}-R_{1}^{2}\right) / \ln \left(R_{2} / R_{1}\right)
\end{aligned}
$$

Where $Q$ is the flow of each controllable chambers $\left(\mathrm{m}^{3} / \mathrm{s}\right) ; N$ is the number of all oil chamber.

In order to simplify the moder of the bearing system, the hydrostatic thrust bearing is assumed that the work table is the ideal smooth surface; the external load is imposed only along the vertical direction, therefore, the worktable occurs the excursion only in this direction, 4 controllable chambers initial flow are the same.

\subsection{Model of Servo Variable Mechanism}

In order to control the flow of the controllable chambers and the oil film thickness, the volume of variable pump is adjusted by the electro-hydraulic servo valve. The servo variable mechanism includes servo amplifier, electro-hydraulic servo valve, servo hydratico cylinder, slanting plate of variable pump and position sensor etc.

The mathematical model of variable pump control mechanism is established according to hydraulic control theory. The following assumptions are carried on in order to simplify analysis:

First, Neglect the influence of the hydraulic oil inertia to the dynamic characteristic of the system, and the fluid is treated as Newtonian fluid; Second, the hydraulic pump leakage is a stable state, neglect the influence of the flow pulsation to the dynamic characteristic of the system; Third, all springs are the linear spring in the system, response lag does not consider. 
1) The transfer function of the servo valve

In servo system, the transfer function expression of the servo valve is determined by system frequency bandwidth.

$$
G_{v}(s)=K_{v} /\left[A\left(T_{v} s+1\right)\right], \quad\left(\omega_{m}<50 H z\right)
$$

Where $K_{v}$ is servo valve gain; $T_{v}$ is the time constant of the servo valve (s); $A$ is the hydraulic cylinder area $\left(\mathrm{m}^{2}\right)$;

2) The transfer function of the Hydraulic cylinders to valve displacement

Because the load of hydraulic cylinder controlling the variable pump slanting plate is relatively small, the fluid leakage and compressibility and the slanting plate reaction are not considered.

$$
\begin{aligned}
& G_{1}(s)=x_{p}(s) / x_{v}(s)=\left(K_{q} / A\right) / S\left(s^{2} / \omega_{h}^{2}+2 \xi_{h} / \omega_{h}+1\right) \\
& \omega_{h}=\sqrt{\left(4 \beta e A^{2}\right) / V_{t} M_{t}} \\
& \xi_{h}=\left(K_{c e} / A\right) \sqrt{\beta e M_{t} / V_{t}}+\left(B_{P} / 4 A\right) \sqrt{\sqrt{\beta e M_{t}}}
\end{aligned}
$$

Where $K_{q}$ is flow $\operatorname{gain}\left(\left(\mathrm{m}^{3} / \mathrm{s}\right) / \mathrm{m}\right) ; \omega_{h}, \xi_{h}$ are respectively the free frequency $(\mathrm{rad} / \mathrm{s})$ and the damping coefficient of the servo-system.; $\beta e$ is the volume spring modulus of the fluid $(\mathrm{Pa}) ; V_{t}$ is the total volume of the hyatic cylinder $\left(\mathrm{m}^{3}\right) ; M_{t}$ is the load quality $(\mathrm{Kg}), K_{c e}$ is the hydraulic cylinder leakage coefficient $\left(\left(\mathrm{m}^{3} / \mathrm{s}\right) / \mathrm{Pa}\right) ; B_{P}$ is the viscous-damping coefficient (N/(m/s)).

3) The relation of displacement of cylinder piston and inclination angle of pump slanting plate:

Where $R$ is the slanting plate radius $(\mathrm{m})$.

4) The relation of the flow of variable pump and inclination angle of slanting plate:

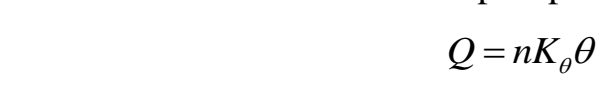

Where $n$ is rotational gpeed of the motor $(\mathrm{r} / \mathrm{s}) ; K_{\theta}$ is the coefficient of volume $\left(\mathrm{m}^{3} / \mathrm{r}\right)$.

5) The transferfunction of displacement sensor:

$$
U_{f}=K_{f} x_{p}
$$

Where $H$ is feedback signal of the displacement (V); $K_{f}$ is the electricity eddy sensor coeflicient $(\mathrm{V} / \mathrm{m})$.

6) The Transfer Function of servo amplifier:

$$
I(s)=K_{a} U(s)
$$

Where $I$ is the amplifier output current (A); $K_{a}$ is the servo amplifier gain (A/V); $U$ is the signal of the input voltage (V). 


\section{Kinematics Relations Equation}

\subsection{Pose and Controllable Chamber Flow Relations}

Worktable origin of coordinates (center of gravity) translates from given position to certain pose because of effect of worktable hot load, cutting force, and rotates along every coordinate axis to new position, $[R]=\left[R_{z}\right]\left[R_{y}\right]\left[R_{x}\right]$ is the transformation matrix[11]. When the worktable is in the random pose (time $t$ ), the coordinates of worktable center point $P\left(x_{p}, y_{p}, z_{p}, \alpha, \beta, \gamma\right)$ changes in the static coordinate system from given pose. Each chamber oil film thickness correspondingly changes. Then the flow of each controllable chamber changes to resist the change of point $P$.

\subsection{Pose Negative Solution}

Each chamber film thickness vector is obtained by the worktable center position pose.

$$
h_{i}=\overrightarrow{O B_{i}}-\overrightarrow{O P}-[R] \overrightarrow{P A_{i}} \quad(i=1,2,3,4)
$$

Where $h_{i}$ is $i$ th oil chamber film thickness vector, $h_{i}=\overrightarrow{\mathrm{PB}}_{i}$ is corresponding the hydraulic cylinder length vector; $\overrightarrow{O P}$ is the wortable center vector, which is measured in the static coordinate system; $\overrightarrow{P A}_{i}$ is contact center vector of the oil chamber film in the worktable, which is measured movement coordinate system, whose value is constant vector; $\overrightarrow{O B_{i}}$ is the controllable chanber displacement vector in the base, which is measured in static coordinate system $[R]$ is pose transformation matrix from movement coordinate system to static coordinate system.

\subsection{Pose Positive Solution Compensation}

The worktable pose positive solution compensation, that is, the actual displacement of controllable chamber film thickness is measured by displacement sensor, via pose positive solution, actual pose of worktable is gained, which is feedback to input to correct error between yorktable desired movement and practical motion, to improve system precision and dynamic quality. Newton-Taylor spread method is used to nonlinear equation in pose positive solution. Because the worktable moves generally nearby in the expected position, initial value of positive solution is set for the worktable in the expected position in the algorithm, that guaranteed algorithm convergence, and the convergence rate will be quicker. Jacobi matrix is used to linearization in the expected position. The linearized kinematics negative solution equation can be expressed as $\Delta l=J \bullet \Delta x$. Where $\Delta l$ is change of controllable chamber film thickness comparing with the expected position; $\Delta x$ is change of worktable pose comparing with the expected position. The linearized kinematics positive solution equation can be expressed

$$
\Delta x=J^{-1} \bullet \Delta l
$$




\section{Worktable Pose Control}

Worktable pose control model is shown in Figure 4. The setting value of film thickness is obtained by the kinematics negative module solution of the given pose; Worktable movement is droved by hydraulic servo system; The worktable pose is described by the change of oil film thickness; The error between film thickness setting value and the measured value enters the controller; The worktable center actual pose is obtained by the kinematics positive solution of four controllable chambers film thickness, compares with the given standard pose, and error enters the controller. This is big closed-loop control. The plant under control is electro-hydraulic servo valve input. The electro-hydraulic servo valve control two-chamber flow of hydraulic cylinder, then flow of controllable chamber and film thickness.

\section{Bearing Pose Control Experiment}

Simulation experiment is carried out for hydrostatic worktable film thickness control system. Given film thickness optimum yalue $h$ is $0.15 \mathrm{~mm}$, then the step input value is $0.15 \mathrm{~mm}$, the step time is 1 second. Model was simulated in Matlab/ Simulink environment, the fixed step was set $0.001 \mathrm{~s}$, the sampling frequency $1000 \mathrm{~Hz}$ was selected and the algorithm ode 45 was applied to the calculation process.

Hardware-in-loop simulation system can permit physical object (servo control chamber) to participate in simulation experiment, which is not easy to model, that can avoid difficulty in modeling. This enhanced the reliability and confidence of simulation result, can simulate various bearing load process in different work condition, the different parameter of thrust bearing and the different type chambers, can satisfy simulation test request in different work condition and different running parameter[12].

In order to verify effectiveness of mathematical model and pose model of bearing system in practical applications, the hardware-in-the-loop simulation test is carried out in valve- ontrol cylinder electro-hydraulic position servo test bench, shown in Figure 5.
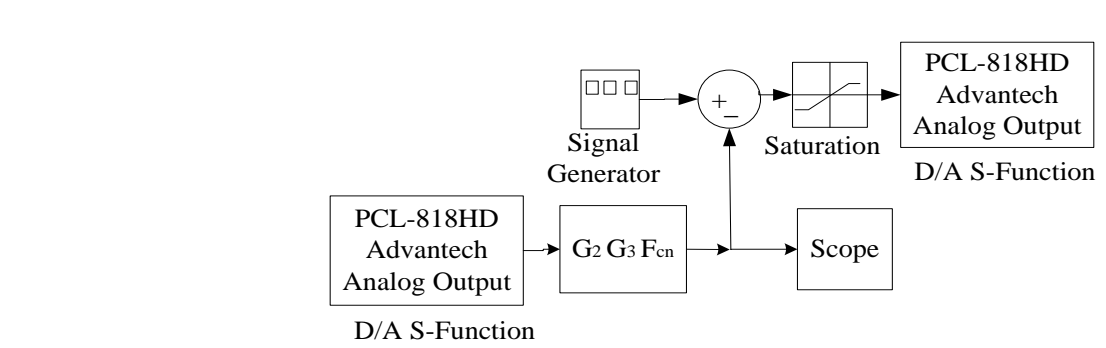

Figure 5. Model of Hardware-in -the-loop Simulation 
The oil film thickness value is acquired by measuring hydraulic cylinder displacement. Thus, effectiveness of bearing system in practical application can be verified by experimenting on electro-hydraulic test.

The disturbance signal is added into system according to change load, cutting force, thermal reaction and system itself parameter change. When the total loads of worktable self weight, work piece weight and the vertical cutting force were considered the bearing worktable strength distortion and the worktable thermal deformation caused by shear fever, which were attained by the finite element analysis software ANSYS computation, were imported to simulation environment. The given pose is $X, X=[0,0,0.15,0,0,2 \pi n]$, where $n$ is rotational speed of spindle.

Computer simulation curve as shown in Figure 6, the step response curve realizes localization to given input signal better. The curve shows that the rising time is $0.096 \mathrm{~s}$, settling time is $0.2724 \mathrm{~s}$ (permissible error range is $2 \%$ ) corrected by controller, response time meets plant demand completely.

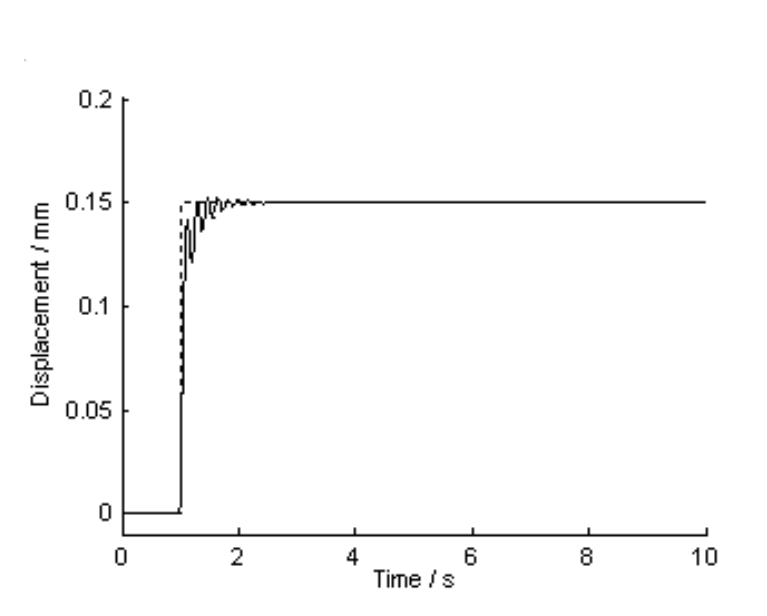

Figure 6. Step Response Curves

The unit step response curve of hardware-in-loop simulation test bench are described in Figure 7, hardware-in loop simulation results show that the rising time is $0.24 \mathrm{~s}$, settling time is $0.4 \mathrm{~s}$ (permissible error range is $2 \%$ ), the maximum overshoot is $2.23 \%$. The practical experimental results by and large accord with the simulation.

\section{Conclusions}

The bearmg worktable pose control was proposed to increase the rigidity of hydrostatic thrust bearing. The controllable chamber flow was adjusted by the valve-control pump variable mechanism, and the flow model was established. The pose control scheme was des gned with the aid of kinematics positive and negative solution. Simulation results show that worktable pose model is reasonable and pose control strategy is effective.

\section{Acknowledgements}

This work was supported by National Natural Science Foundation of China (51075106, 51005063, 50975066) and Technology item of Heilongiang Provincial Education Department (No.11531051, No.11551080). 


\section{References}

[1] S. C. Sharama, S. C. Jain and D. K. Bharuka, "Influence of Recess Shape on the Performance of a Capillary Compensated Circular Thrust Pad Hydrostatic Bearing", Tribology International, vol. 35, (2002), pp. 347356.

[2] T. A. Osman, Z. S. Safar and M. O. A. Mokhtar, "Design of Annular Recess Hydrostatic Thrust Bearing under Dynamic Loading", Tribology International, vol. 24, (1991), pp. 137-141.

[3] R. E. Johnson and N. D. Manring, "Sensitivity studies for the shallow-pocket geometry of a hydrostatic thrust bearing", American Society of Technology Division FPST, vol. 10, (2003), pp. 231-238.

[4] S. C. Sharama, S. C. Jain and D. K. Bharuka, "A Comparative Study of Flexible Thrust Pad Hydrostatic Bearings with Different Restrictors", Wear, vol. 121, (1988), pp. 53-70.

[5] V. K. Kapur and K. Verma, "The Simultaneous Effects of Inertia and Temperature on the Performance of a Hydrostatic Thrust Bearing”, Wear, vol. 54, (1979), pp. 113-122.

[6] C. K. Singh and D. V. Singh, "Stiffness Optimization of a Variable Restrictor-Compensated Hydrostatic Thrust Bearing", Wear, vol. 44, no. 2, (1977), pp. 223-230.

[7] T. Jayachandra Prabhu and N. Ganesan, "Behaviour of Multirecess Plane Hydrostatic Thrust Bearings under Conditions of Tilt and Rotation”, Wear, vol. 92, (1983), pp. 243-251.

[8] T. Jayachandra Prabhu and N. Ganesan, "Effect of Tilt on the Characteristics of Mul ireces Hydrostatic Thrust Bearings under Conditions of no Rotation", Wear, vol. 92, (1983) pp 269-277.

[9] W. Feng, Y. Jun-yi and L. Long, "Hydraulic and automatic leveling control system of vehicle-mounted anti aircraft gun", Journal of Gun Launch \& Control, vol. 1, (2007), pp.67-71.)

[10] D. Zhengqian, "Design of liquid bearing: Shanghai Science Technique Press", Shanghâi, (1989).

[11] X. Ke-qiang, Y. Xian-wan and W. Ji-kun, "Kinetic study on pressure leaching of high iron sphalerite concentrate", Transactions of Nonferrous Metals Society of China, vol. 17, (2007), pp. 187-194.

[12] F. Rune, K. Rao Sudhakara and A. Joran, "Real time hardware-in-loopsimulation of ESMO satellite attitude control system", Modeling, Identification and Control, vol. 27, (2006), pp 125-140.

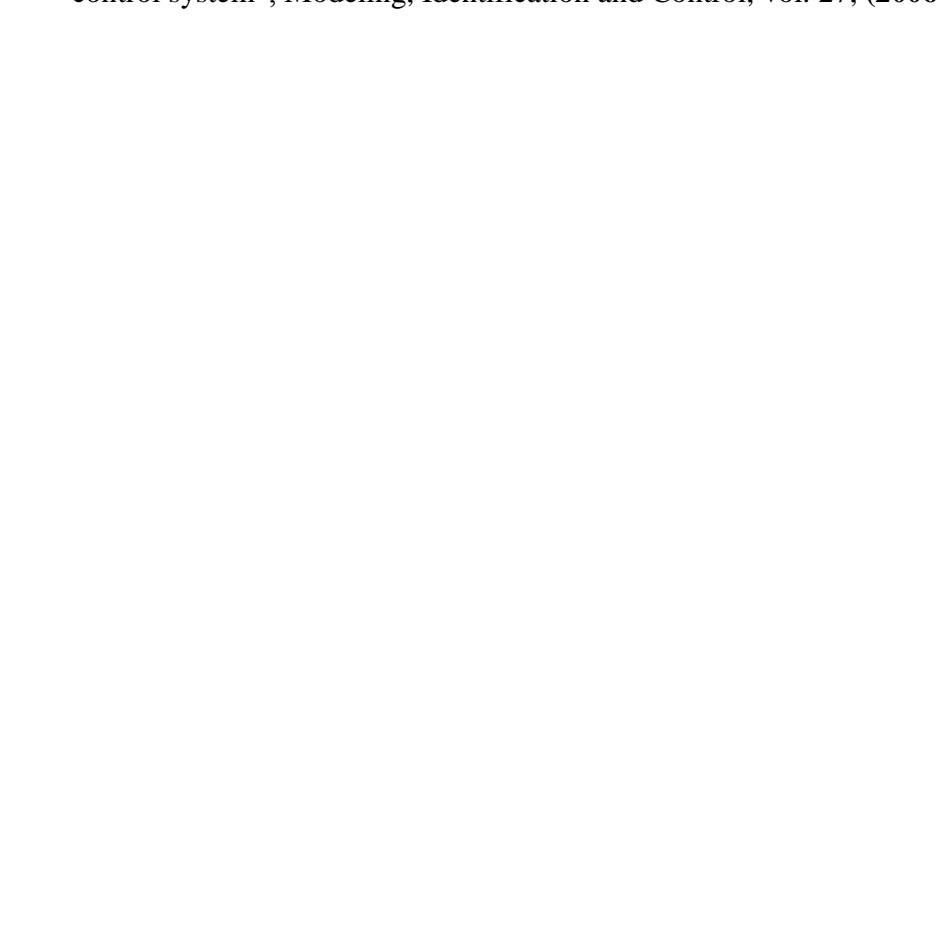


International Journal of Smart Home

Vol.8, No.1 (2014)

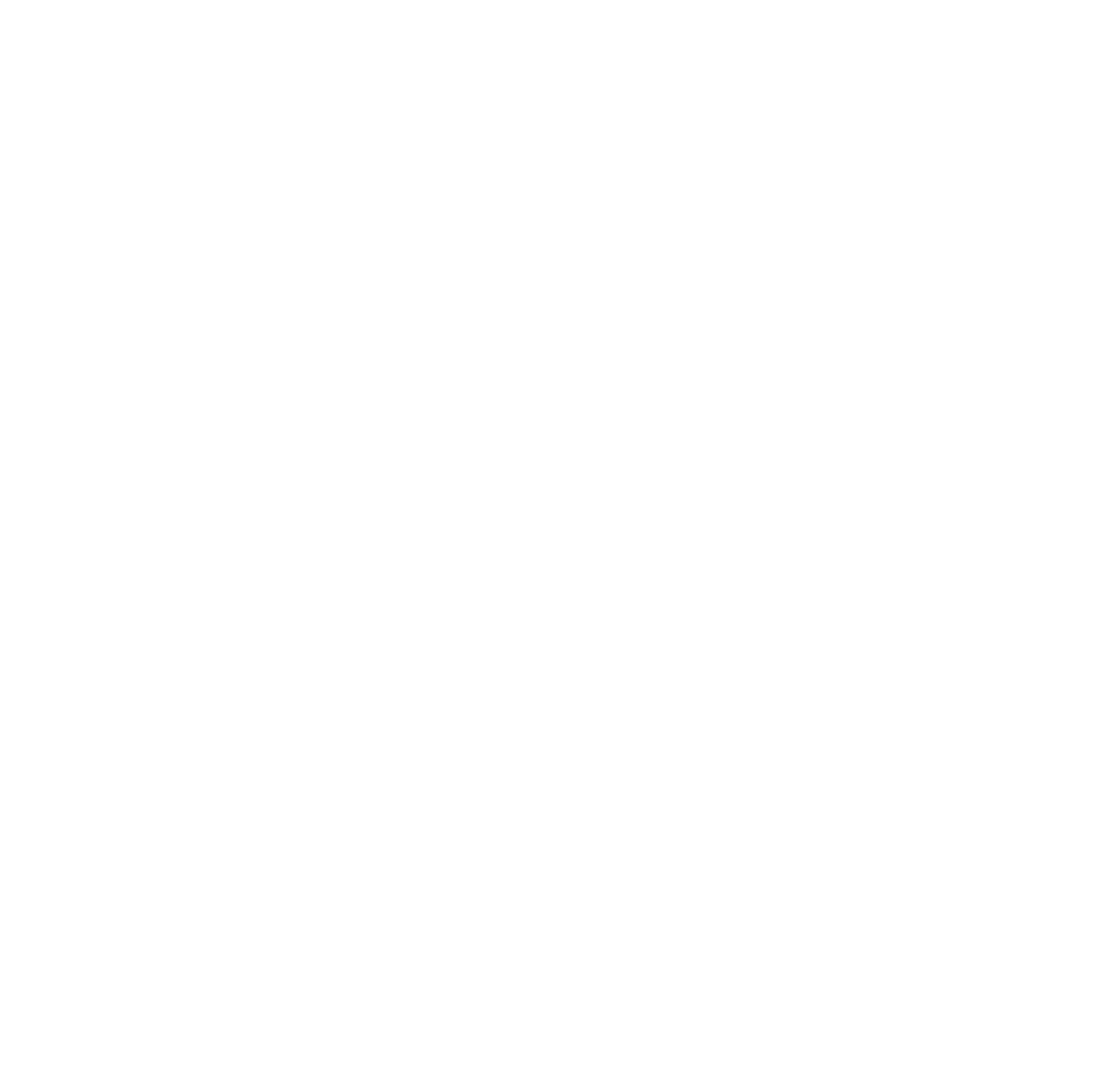

